\title{
Optimal mix of renewable power generation in the MENA region as a basis for an efficient electricity supply to europe
}

\author{
Alaa Alhamwi ${ }^{a}$, David Kleinhans, Stefan Weitemeyer and Thomas Vogt \\ NEXT ENERGY - EWE Research Centre for Energy Technology at the University of Oldenburg, \\ Carl-von-Ossietzky-Str.15, 26129 Oldenburg, Germany
}

\begin{abstract}
Renewable Energy sources are gaining importance in the Middle East and North Africa (MENA) region. The purpose of this study is to quantify the optimal mix of renewable power generation in the MENA region, taking Morocco as a case study. Based on hourly meteorological data and load data, a 100\% solar-plus-wind only scenario for Morocco is investigated. For the optimal mix analyses, a mismatch energy modelling approach is adopted with the objective to minimise the required storage capacities. For a hypothetical Moroccan energy supply system which is entirely based on renewable energy sources, our results show that the minimum storage capacity is achieved at a share of $63 \%$ solar and $37 \%$ wind power generations.
\end{abstract}

\section{Introduction}

The electricity supply systems in the MENA region are currently facing challenges such a substantial increase of the electricity demand over the next decades as well as the global issue of climate change [1]. Hence, a strong fundament for a secure, environmental friendly and economically efficient energy supply system is needed in the region. MENA has about $70 \%$ of the world's proven oil reserves and $50 \%$ of the proven gas reserves [2], its energy system infrastructure today depends almost entirely on fossil fuels. On the other hand, the region is also a unique place for harnessing renewable energies at large scales as $45 \%$ of the world's potential for solar energy is concentrated in the MENA deserts [3]. In addition, there is a strong potential for wind energy [4].

The MED-CSP study states that "A well balanced mix of renewable energies can replace electricity from fossil fuels" [4]. Moreover, the ENCOURAGED ${ }^{1}$ project illustrates that $15 \%$ to $20 \%$ of the total EU-27 electricity demand by 2050 could be covered by imports of renewable electricity from countries of the MENA region [5, 6]. However, in order to approach a stable, ecological friendly and economically efficient power supply system with the potential to export renewable electricity from the MENA region to Europe, it is important to first quantify the optimal mix of different renewable power technologies on

\footnotetext{
a Corresponding author: alaa.alhamwi@next-energy .de

${ }^{1}$ ENCOURAGE project aimed at finding the most economical energy connections for electricity, natural gas and hydrogen between Europe and the neighbouring region such as MENA region http://ec.europa.eu/research/fp6/ssp/ encouraged_en.htm.
}

This is an Open Access article distributed under the terms of the Creative Commons Attribution License 4.0, which permits unrestricted use, distribution, and reproduction in any medium, provided the original work is properly cited. 
the basis of the long-term meteorological conditions to enable efficient and cost-effective planning of power supply systems in the region. Therefore, this study quantifies the optimal mix of renewable power generation capacities in a $100 \%$ renewable energy scenario which leads to a minimal need of storage capacities. For this purpose, we analysed specifically meteorological data and load data for Morocco. The country has been chosen due to its representative character for the MENA region and due to the sufficient availability of data.

\section{Data sources and power mix}

The main inputs of the model are long-term meteorological and load data of Morocco. Hourly time series of meteorological parameters like solar irradiation and wind speed as well as load data are collected from different sources and integrated into a database. Afterwards the meteorological data are converted to power output. The processing of the model input data is described in chapters 2.1 to 2.4 below.

\subsection{Solar irradiance data}

Direct Normal Irradiation (DNI), Diffused Horizontal Irradiance (DHI) and Global Horizontal Irradiance (GHI) values are taken from the Solar Radiation Data (SoDa) database for the year 2005. This solar radiation database is estimated from Meteosat images [7]. The locations of solar power plants are chosen according to the Moroccan Solar Plan (MSP) which intends to install a total of $2.000 \mathrm{MW}$ solar power by 2020 [9] in the following locations: Ouarzazate site, Ain Beni Mathar site, Foum Al Ouad site, Boujdour site, and Sebkhat Tah site. Morocco has currently 20 MW CSP installed capacity in the municipality of Ain Beni Mathar and $160 \mathrm{MW}$ under construction in the municipality of Ouarzazate. It has also about $15 \mathrm{MW}$ installed capacity PV in operation [10].

SoDa provides these data at an hourly resolution. The database has few missing data which have been interpolated linearly. The data are converted to TMY3 ${ }^{2}$ files in order to be used for the System Advisory Model (SAM) software [8]. From these data a time series of solar electricity power $\mathrm{S}(\mathrm{t})$ is calculated using SAM.

\subsection{Wind data}

Wind speed data for the year 2005 are provided by the European Centre for Medium-Range Weather Forecasts (ECMWF) which is located in the UK and the Centre for Wind Energy Research (ForWind) at the University of Oldenburg in Germany [11]. The data are available for intervals of six hours. All wind data are interpolated linearly to an hourly resolution to be comparable with the solar and load data sets used in this work. Linear interpolation has been found to be the most appropriate method for the specific data comparing to other interpolation methods [12].

In order to estimate the wind power output $\mathrm{W}(\mathrm{t})$ from the wind speed data the following equation is used:

$$
\mathrm{W}(\mathrm{t})= \begin{cases}\mathrm{P}_{\mathrm{r}}\left(\frac{\mathrm{v}^{3}(\mathrm{t})}{\mathrm{v}_{\mathrm{r}}^{2}}\right) \text { if } \quad \mathrm{v}_{\text {in }} \leq \mathrm{v}(\mathrm{t}) \leq \mathrm{v}_{\mathrm{r}} \\ \mathrm{P}_{\mathrm{r}} & \text { if } \quad \mathrm{v}_{\mathrm{r}} \leq \mathrm{v}(\mathrm{t}) \leq \mathrm{v}_{\text {out }} \\ 0 & \text { otherwise }\end{cases}
$$

\footnotetext{
2 Typical Meteorological Year, version 3 (TMY3) file format required by SAM software.
} 
$\mathrm{W}(\mathrm{t})$ represents the time series of wind power generation, $\mathrm{P}_{\mathrm{r}}$ is the rated power, $\mathrm{v}(\mathrm{t})$ wind speed at time $t, v_{\text {in }}$ is the cut-in speed, $v_{r}$ is the rated speed and $v_{\text {out }}$ is the cut-out speed. The values of $v_{\text {in }}, v_{r}$ and $\mathrm{v}_{\mathrm{out}}$ are taken from a wind power curve of a representative wind turbine $\left(\mathrm{v}_{\text {in }}=4 \mathrm{~m} / \mathrm{s}, \mathrm{v}_{\mathrm{r}}=13 \mathrm{~m} / \mathrm{s}\right.$, $\mathrm{v}_{\text {out }}=25 \mathrm{~m} / \mathrm{s}$ and $\mathrm{P}_{\mathrm{r}}=1.5 \mathrm{MW}$, taken from the SAM Library). The electric power output is calculated for the following five locations, where wind farms projects are already under operation, planned or under construction [10, 13]: Tetouan site, Essaouira site, Tantan site, Tarfaya site and Laayoune site. Morocco has today wind turbines with an installed capacity of $339 \mathrm{MW}$. In addition, $600 \mathrm{MW}$ are currently under construction [10].

\subsection{Load data}

A complete time series of hourly load data for Morocco for the year 2010 is obtained from the Office of National Electricity (ONE) [14].

\subsection{Other weather data}

The hourly weather data for ambient temperature, pressure and wind direction in the year of 2005 are provided on a six-hourly resolution and are based on ECMWF data. They are also provided by ForWind. The relative humidity is calculated applying the August-Roche-Magnus approximation [15]. Weather data are interpolated linearly and combined with the solar irradiance and wind data to create TMY3 files to be compatible with SAM [8].

\section{Model overview}

Renewable power generations like solar and wind have a fluctuating and variable power output. Therefore, there is need for energy storages in the future energy systems in order to balance between renewables power supply and demand. In this study, we investigate the optimal combination of solar and wind power with respect to the minimum required storage capacities. Heide et al. used the mismatch energy concept in order to quantify the optimal mix of renewable power sources in a specific region $[16,17]$. The concept illustrates the difference between supply and demand at any time.

Equation (2) represents the time series of mismatch energy between the hourly wind and solar power generations and the hourly load.

$$
\Delta(t, a, b)=\gamma\left(a \frac{\mathrm{W}(t)}{<\mathrm{W}>}+b \frac{\mathrm{S}(t)}{<\mathrm{S}>}\right)-\frac{\mathrm{L}(t)}{<\mathrm{L}>} ; \quad \gamma>1
$$

- $\Delta(t, a, b)$ : represents the time series of mismatch energy as a function of time and the coefficients $a$ and $b$,

- $a, b$ : are coefficients that show how much of the load is on average covered by wind and solar power generation. The sum of both coefficients is constrained to $100 \%$, i.e. $a+b=1$.

- $\gamma$ : is the excess or surplus generation factor,

- $\mathrm{W}(\mathrm{t})$ : is the total wind power generation during time $\mathrm{t}$,

- $\mathrm{S}(t)$ and $\mathrm{L}(t)$ are the solar power and load power generation time series, respectively,

- $<>$ the average over all time $t$,

In order to calculate the coefficients at which the optimal combination between renewable power generations is defined, Heide et al. present a simplified storage model approach which aims to define the optimal coefficients values at which the required balancing energy and the storage capacity reach its minimum. 
In Eq. (3) $\mathrm{H}(t, a, b)$ represents the time series of the storage filling level as a function of time and the coefficients $a$ and $b$ :

$$
\mathrm{H}(t, a, b)=\mathrm{H}(t-1, a, b)+\left\{\begin{array}{l}
\eta_{\text {in }} \Delta(t, a, b) \text { if } \Delta(t, a, b) \geq 0 \\
\eta_{\text {out }}^{-1} \Delta(t, a, b) \text { if } \Delta(t, a, b)<0
\end{array}\right.
$$

If the mismatch energy at time $\mathrm{t}$ in Eq. (3) is positive, the excess energy will be stored with an efficiency $\eta_{\text {in }}$. If the mismatch energy at time $t$ is negative, the storage is discharged with an efficiency $\eta_{\text {out }}$. The maximum difference between the filling level at time $t$ and the minimum filling level at time $t^{\prime}$ determines the required storage size Hence, $\mathrm{E}_{\mathrm{H}}$ in Eq. (4) determines the minimum required storage at a specific combination of renewable power generation.

$$
\mathrm{E}_{\mathrm{H}}(a, b)=\max _{t}\left(\mathrm{H}(t, a, b)-\min _{t^{\prime} \geq t} \mathrm{H}\left(t^{\prime}, a, b\right)\right) .
$$

In summary, it can be stated that for the calculation of the optimal mix, it is necessary:

- to create an integrated hourly time series of weather and load data,

- to convert meteorological data to power production using solar and wind power generator technologies and

- to calculate the optimal mix and the required storage according to Eqs. (2) to (4).

Our model is implemented in the programming language Python.

\section{Results - The optimal power generation mix}

It is very likely that solar and wind power will be of major importance for the future electricity supply of the MENA region. Therefore, this study investigates a hypothetical 100\% solar-plus-wind scenario for a potential future power supply of Morocco as a representative country of the MENA region. For the quantification of the optimal mix of solar and wind power generations, we calculated the required storage for different combinations of wind and solar power, i.e. at different values of a and b coefficients, as shown in Eq. (2). The optimal mix comes at the minimum required storage. In this study, the optimal mix is calculated for two cases: for an idealised storage without any losses and a typical storage with a charging efficiency $\eta_{\text {in }}$ and discharging efficiency $\eta_{\text {out }}$.

For the case of an ideal storage without any losses $\left(\eta_{\text {in }}=\eta_{\text {out }}=100 \%\right)$ and $\gamma=1.1$, the optimal mix of solar and wind in Morocco is found $a t b=0.82$ and $a=0.18$. This result means that the solar power generation should cover $82 \%$ of the demand and the remaining share of $18 \%$ is provided by wind power. However, an ideal storage is not a realistic option since the conversion of energy is always associated with losses. For example, current pumped-hydro storage efficiencies are in the order of $\eta_{\text {in }}=\eta_{\text {out }}=90 \%$ [16]. For comparison, future promising storage technologies which are still in their development phase or at least optimisation phase like hydrogen show efficiencies of $\eta_{\text {in }}=\eta_{\text {out }} \approx 60 \%$ [16].

Morocco has a potential for pumped-hydro storage. It currently has a $465 \mathrm{MW}$ pumped-hydro power station located at Afourer, Tadla-Azilal [18]. In this study a pumped-hydro storage with efficiencies of $\eta_{\text {in }}=\eta_{\text {out }}=90 \%$ [16] is chosen as a storage technology. Hence, the solar and wind power generation have to increase in order to compensate the storage losses. A surplus generation factor of $\gamma=1.1$ is taken in this study, which means $10 \%$ overproduction of electricity by solar and wind power.

Figure 1 shows a comparison between the required storage in average hourly load (av.h.l) for an idealised storage and a storage with losses at the same value of $\gamma=1.1$. A comparison of both storage types demonstrates that the required storage increases significantly when storage losses are taken into account. In both cases, the best combination of solar and wind power generations is chosen at the minimum required storage.

As shown in Fig. 1 the optimal mix of renewable power technologies with the minimal demand for storage is reached at the combination of $b=0.82$ and $a=0.18$ in case of ideal storage and $b=0.63$ and 


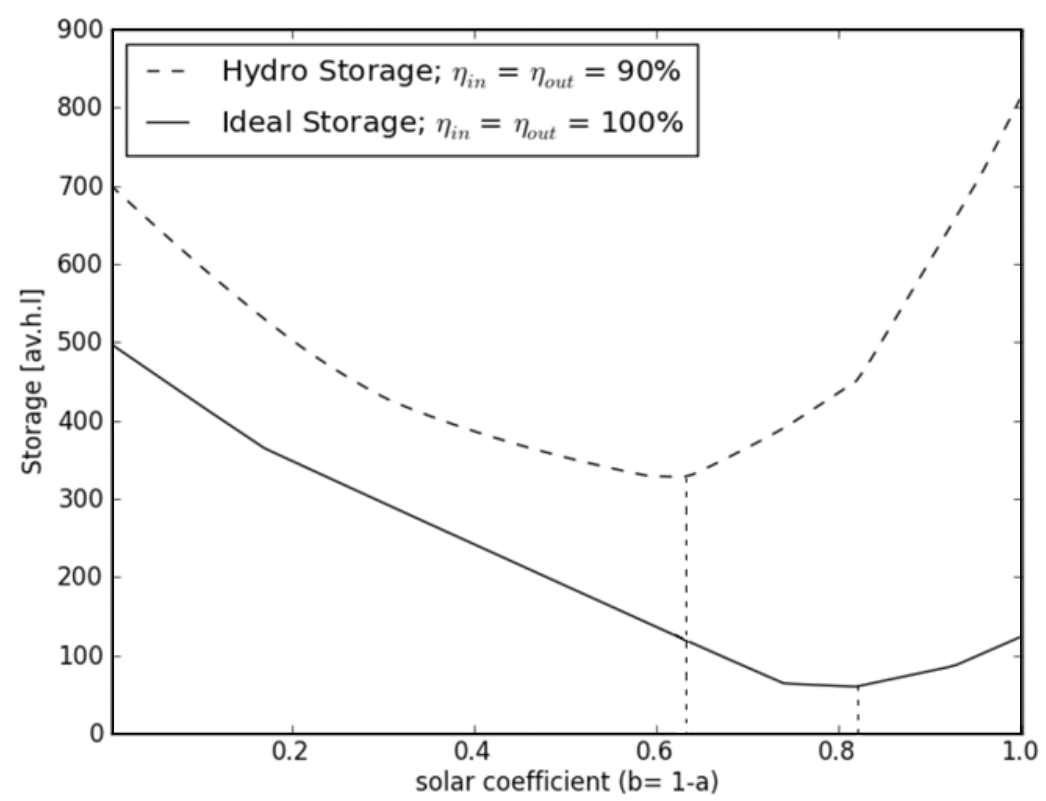

Figure 1. Storage as a function of the solar coefficient $(b=1-a)$ in $100 \%$ only solar and wind scenario in Morocco at $\gamma=1.1$ in two cases: an ideal storage and for a pumped-hydro storage with $\eta_{\text {in }}=\eta_{\text {out }}=90 \%$. The storage unit is in average hourly load (av.h.l).
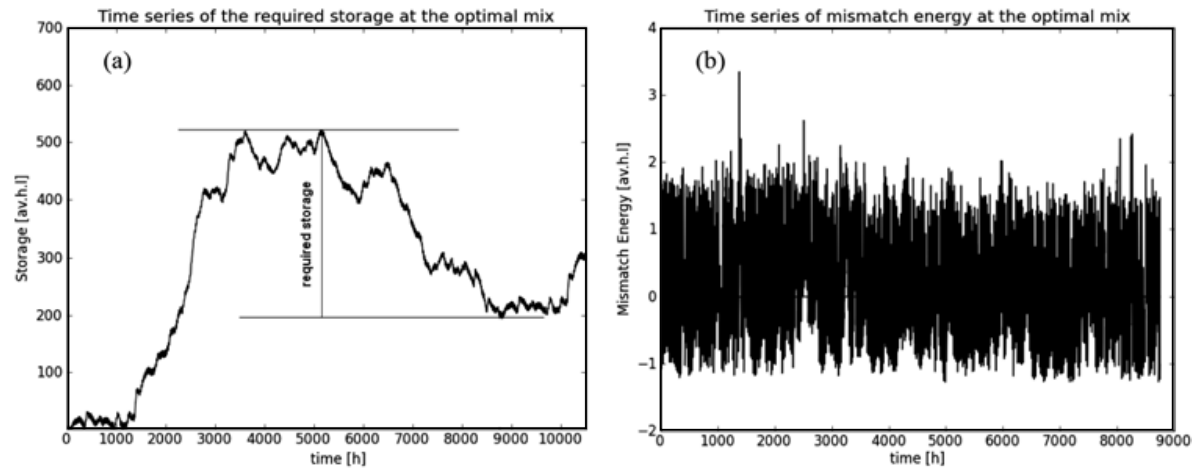

Figure 2. (a) Time series of the storage level for the optimal mix scenario $H(t, a=0.37, b=0.63)$. (b) The corresponding mismatch energy $\Delta(\mathrm{t}, \mathrm{a}=0.37, b=0.63)$ in average hourly load (av.h.l.).

$\mathrm{a}=0.37$ for the pumped-hydro storage. Figure 1 illustrates that for a $100 \%$ only-wind (solar coefficient $b=0$ ) or a $100 \%$ only-solar scenario (solar coefficient $b=1$ ) the required storage increases substantially. In case of hydro storage, for instance, the required storage in a $100 \%$ only-solar scenario is about 800 av.h.l., which is more than twice the required storage at the optimal mix.

In both cases (ideal and pumped-hydro storage) a combination of the renewable power technologies minimises the need of balancing energy and consequently the demand of storage capacity. The required storage for the idealised storage is about 60 av.h.l. and the pumped-hydro storage is approximately 400 av.h.l.

Figure 2a shows a time series of the filling level of the pumped-hydro storage at the optimal mix. The maximum required storage is defined by maximum difference between the filling level at time $\mathrm{t}$ 
and the minimal next one at time t'. In order to avoid spurious effects of the time frame of simulation (one calendar year in this study) on the estimated minimal storage requirements, storage time series for two calendars periods (with periodic boundary conditions for the mismatch time series) need to be considered as shown in Fig. 2a.

The hourly time series of mismatch energy is shown in Fig. $2 b$ for one year. If $\Delta(\mathrm{t}, \mathrm{a}, \mathrm{b})>0$ the storage is charged with an efficiency $\eta_{\text {in }}=90 \%$. The storage is discharged with an efficiency $\eta_{\text {out }}=90 \%$ in case of $\Delta(\mathrm{t}, \mathrm{a}, \mathrm{b})<0$.

\section{Discussion}

Our analysis shows that the calculation of the optimal mix of renewable power sources provides valuable information for the potential design of Morocco's future power supply system and power supply systems in general. For instance, for the investigated hypothetical scenario the annual power production and required installed capacities for each power technology can be approximated from our calculations. Hence, assuming an average annual electricity demand of $28 \mathrm{TWh}$, which is comparable with Morocco's 2010 consumption value, the optimal mix model suggests that $63 \%$ of the average load (18 TWh for the annual consumption of 2010) should be covered by solar. The remaining share would need to be covered by wind $(37 \%)$ which would need to cover $10 \mathrm{TWh}$ of the electricity demand. Hence, the optimal mix model which is described in this paper provides a basis to develop scientific recommendations to policy makers and national energy strategy designers for the development of energy mix strategies to reduce investments in power supply and storage capacities due to a long-term meteorological optimisation.

Due to the dynamic behaviour of renewable power generation, the need for storage is crucial. Pumped-hydro storage is an option and Morocco has a pumped-hydro storage plant [18]. However, alternative storage technologies (e.g. compressed air or hydrogen) also need to be considered. Another suggestion to overcome fluctuations in wind and solar power generation could be a stronger link of the Mediterranean grid interconnections between Europe and the MENA region. In such a scenario, countries of the MENA region could export excess energy to Europe and import electricity in times of deficit power generation.

From a meteorological perspective, the analyses of long-term weather data can have a substantial impact on the design of national power supply systems. The results gained in this paper associated with the required storage capacity could imply less cost compared to other electricity mixes. However, for further work it is crucial to distinguish different storage types, short term or long term, hydropower or hydrogen. Further work should be done for cost analyses as one of the main parameters and also for quantifying the optimal mix between renewable and non-renewable power generation med-term scenarios by finding the optimal mix.

\section{Conclusion}

This work investigates the optimal combination of renewable energy technologies to fulfil Morocco's energy demand in a $100 \%$ only solar-plus-wind scenario at a minimum capacity of energy storage for balancing purposes. Based on meteorological and load parameters, the optimal mix is found to be $63 \%$ solar and $37 \%$ wind power if pumped-hydro storage is used for balancing. However, other parameters need to be investigated such as costs, social and environmental impacts of the optimal electricity mix.

The transition towards a power supply system in the EUMENA region which is entirely based on renewables implies multiple challenges of various dimensions (e.g. technological, economical, ecological and social acceptance). Even more complex, these dimensions are strongly interlinked to each other. Due to the complexity of such systems there is a strong demand for multi-criteria decision making tools that are able to evaluate such scenarios and national energy strategies. 
The authors would like to thank the European Centre for Medium Range Weather Forecast (ECMWF) and the Centre for Wind Energy Research (ForWind) for the supply of weather data. We acknowledge also Office National de l'Electricité (ONE) for providing the hourly load curve data of Morocco.

\section{References}

[1] ERECE, European Renewable Energy Council, RE-thinking 2050, http://www.rethinking 2050.eu/fileadmin/documents/ReThinking2050_full_version_final.pdf (2012)

[2] IIF, The Institute of International Finance (IIF), Economic research, MENA. http://www. iif.com/emr/mena/ (2013)

[3] D. Ramin Jalilvand, Renewable Energy for the Middle East and North Africa Policies for a Successful Transition, http://library.fes.de/pdf-files/iez/08959.pdf (2012)

[4] DLR, German Aerospace Center. Concentrating Solar Power for the Mediterranean Region, http://www.dlr.de/Portaldata/1/Resources/portal_news/newsarchiv2008_1/ algerien_med_csp.pdf (2005)

[5] F. Trieb, M. O'Sullivan, T.Pregger, C. Schillings, W. Krewitt, Characterisationof Solar Electricity Import Corridors from MENA to Europe, http://www . dlr.de/Portaldata/41/Resources/dokumente/institut/system/publications/ Solar_import_DLR_2009_07.pdf (2009)

[6] European Commision, Energy corridors European Union and Neighbouring countries, http://ec.europa.eu/research/energy/pdf/energy_corridors_en.pdf (2007)

[7] Solar Energy Services for Professionals, Solar Radiation Data, http://www.soda-is.com/eng/services/services_radiation_free_eng.php

[8] SAM, The System Advisor Model, https://sam.nrel.gov/

[9] MASEN, Moroccan Agency for Solar Agency, http://www.masen.org.ma/index.php? $\mathrm{Id}=43 \&$ lang=en\#/

[10] RCREEE, Regional Centre for Renewable Energy and Energy Efficiency, Renewable Energy, Country Report Morocco, http://www.rcreee.org/wp-content/uploads/ 2013/05/RCREEE_CountryProfile_RE_Morocco_EN_2012.pdf (2013)

[11] ForWind, The Centre for Wind Energy Research, University of Oldenburg, http://www. forwind.de/

[12] EWEA, European Wind Energy Association, Integrating Wind Developing Europe's power market for the large-scale integration of wind power, http: //www . uwig. org/TradeWind.pdf (2009)

[13] MEMEE, Ministry of Energy, Mines, Water and Environment, http://www . mem.gov .ma

[14] ONE, Office National de l'Electricité, http://www.one.org.ma/

[15] B. McNoldy, http://andrew.rsmas.miami.edu/bmcnoldy/humidity_conversions.pdf (2001)

[16] D. Heide, L. von Bremen, M. Greiner, C. Hoffmann, M. Speckmann, S. Bofinger, Renewable Energy, 35, 2483-2489 (2010)

[17] D. Heide, M. Greiner, L. von Bremen, C. Hoffmann, Renewable Energy, 36, 2515-2523 (2011)

[18] GEO, Global Energy Observatory, http://globalenergyobservatory.org/form. php?pid $=41620$ 